\title{
FINE NEEDLE ASPIRATION BIOPSY OF THYROID NODULES: CYTOHISTOLOGICAL CORRELATION
}

\author{
Siderova M. ${ }^{1}$, K. Hristozov ${ }^{1}$, I. Krasnaliev ${ }^{2}$, R. Radev $^{3}$, R. Nenkov ${ }^{3}$ \\ ${ }^{1}$ Clinic of Endocrinology, ${ }^{2}$ Department of Pathology, ${ }^{3}$ Clinic of Thoracic Surgery, \\ University hospital "St. Marina", Varna, Bulgaria
}

Reviewed by: Assoc. Prof. Hr. Bohchelian, MD, PhD

\begin{abstract}
OBJECTIVE: Fine-needle aspiration biopsy (FNAB) currently is the main procedure for distinguishing benign from malignant thyroid nodules. The aim of our study was to assess the accuracy of FNABs in our hospital by comparing the cytological and histological diagnosis. METHODS: We performed a prospective study including 321 patients with thyroid nodules admitted to "St. Marina" Hospital from January 2004 to December 2006. Clinical and US data, TSH, FT3, FT4, anti-TPO, cytological and histological findings were evaluated. RESULTS: FNABs were performed in all 321 cases, 290 of them were women and 31 men; mean age 52,9 years. Cytological evaluation considered $67,3 \%$ of FNABs as benign, $15,6 \%$ as suspicious, $4,7 \%$ as malignant and $12,4 \%$ as inadequate sample. 88 of the patients underwent thyroid surgery. The comparison between cytological and histological findings showed that $99,1 \%$ of cytologically benign nodules were histologically confirmed, as well as $100 \%$ of the malignant lesions. $89,5 \%$ of the cases classified as suspicious on FNAB turned out to be benign on histology and $10,5 \%$ were malignant. The discrepant cases were 2 false-negative results, which had a cytological diagnosis of nodular hyperplasia and turned out to be a medullary carcinoma. Our results showed a sensitivity of $\mathbf{8 8 , 2} \%$ and a specifity of $100 \%$. CONCLUSION: Thyroid FNAB is an accurate and cost-effective preoperative tool for selection of patients who would benefit from surgery.
\end{abstract}

Keywords: thyroid nodules, FNAB, malignancy, surgery

\section{INTRODUCTION}

Nodular thyroid disease is found in approximately $5 \%$ of the population at palpation screening and is almost ten-fold more frequent at ultrasound screening. Malignant nodules are rare and account for $5 \%$ of all nodules. Fine-needle aspiration biopsy (FNAB) has now gained wide acceptance as the most accurate diagnostic procedure for distinguishing benign from malignant nodules.

The aim of this study was to assess the accuracy of FNABs in our hospital by comparing the cytological and histological diagnosis and to identify the limitations of FNAB in the management of thyroid nodule disease in North-Eastern Bulgaria.

\section{MATERIALS AND METHODS}

We performed a prospective study including 321 patients with thyroid nodules admitted to "St. Marina" Hospital from January 2004 to December 2006. We evaluated the clinical and US

Address for correspondence:

Mira Siderova, MD, Clinic of Endocrinology, University hospital

"St. Marina", Varna 9010, "Hr. Smirnenski"str. 1, Bulgaria,

E-mail:mirasiderova@abv.bg data (9 MHz; Color Doppler), scintigraphy, TSH, FT3, FT4, anti-TPO. US-guided FNABs with cytological examination were performed in all 321 cases of nodular thyroid disease. All malignant and indeterminate (suspicious) lesions, as well as hot benign nodules and larger than $3 \mathrm{~cm}$ nontoxic benign nodules were referred to surgery with histological evaluation by two independent pathologists.

Statistical analysis was carried out using SPSS ver.11.0 and MS Excel software programs.

Sensitivity, specificity, positive and negative predictive value, and diagnostic accuracy of FNAB were assessed as follows. Histological diagnosis was considered the gold standard. Sensitivity was defined on the basis of thyroid malignant lesions detected by FNAB and confirmed by histology (true positive/ true positive + false negative). Specificity was defined on the basis of benign nodules detection (true negative/ true negative + false positive). Diagnostic accuracy was calculated as (true positive + true negative)/ (true positive + false positive + true negative + false negative).

\section{RESULTS}

FNABs were performed in all 321 cases included in our study, $290(90,3 \%)$ of them were women and $31(9,7 \%)$ 
men; mean age 52,9 $\pm 14,1$ years (fig. 1 ). The mean nodule volume was $4,3 \mathrm{ml}$. The majority of nodules were smaller than $2 \mathrm{ml}(58 \%)$ and were either solid hypoechogenic or mixed with areas of cystic degeneration (fig.2 and fig.3).

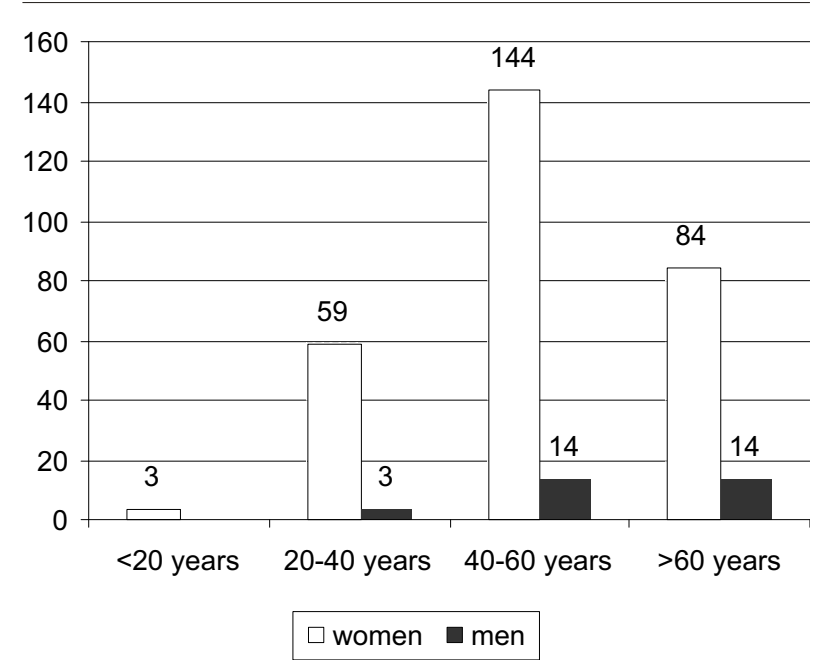

Fig. 1 Distribution of thyroid nodules in different age groups among women and men

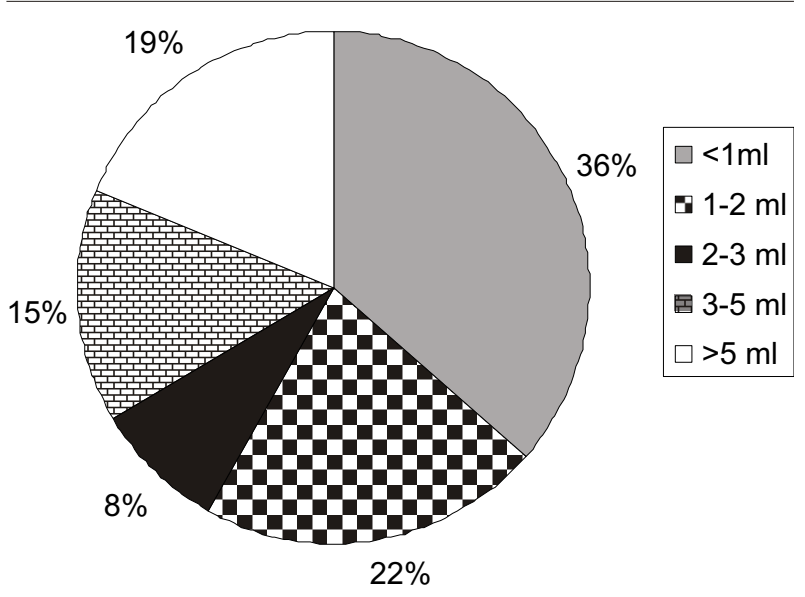

Fig. 2 Thyroid nodule volume

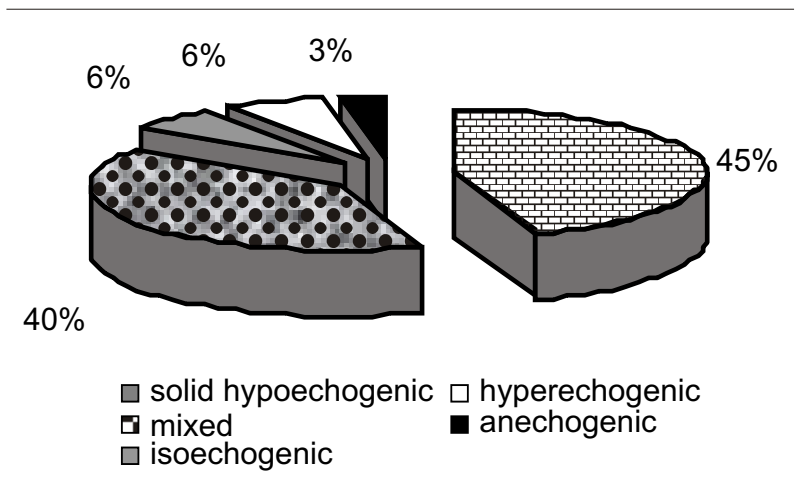

Fig. 3 Distribution of thyroid nodules according to US characteristics

Cytological evaluation considered $67,3 \%$ of FNABs as benign - nodular goiter 39,9\%, adenomatoid nodules $18,1 \%$, lymphocytic thyroiditis $8,7 \%$ and granulomatous thyroiditis $0,6 \%$. Indeterminate or suspicious were $15,6 \%$ of the nodules
- follicular neoplasma 11,2\%, Hurtle cell neoplasma 3,5\%, suspicious medullary neoplasma $0,3 \%$ and others $0,6 \%$. Cytology assessed $4,7 \%$ of the nodules as malignant - papillary carcinoma $1,6 \%$, follicular carcinoma $0,7 \%$, anaplastic carcinoma $0,6 \%$, malignant lymphoma $0,6 \%$, Hurtle cell carcinoma $0,3 \%$, metastatic carcinoma $0,9 \%$. Inadequate samples comprised $12,4 \%$ of the FNABs. (fig.4).

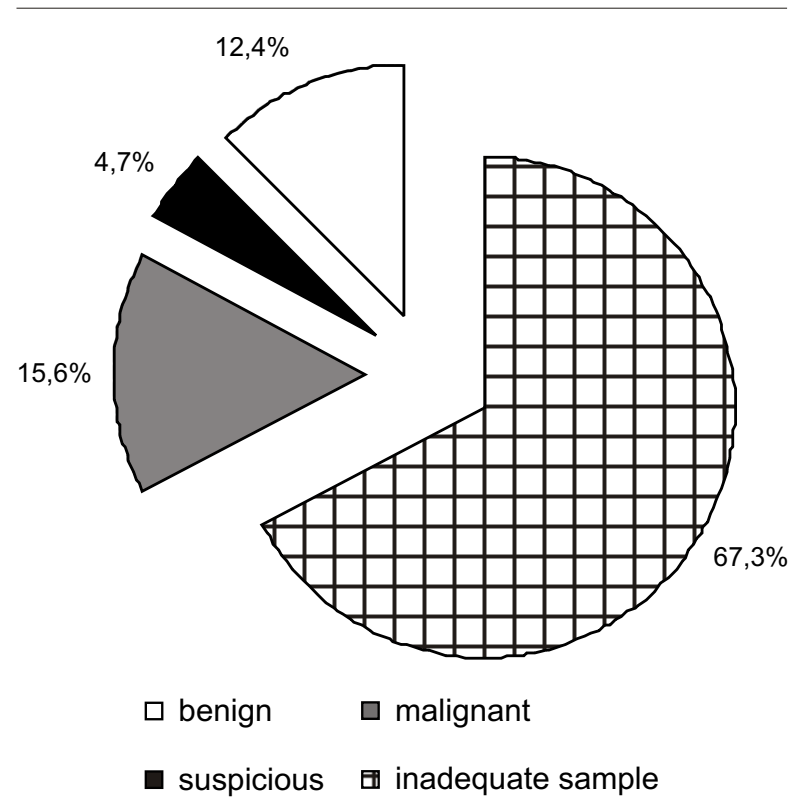

Fig. 4 Results from cytological evaluation of nodules after FNAB

Correlation between nodule echogenicity and cytological result after FNAB confirmed

that most of the malignant and suspicious nodules are hypoechogenic, whereas anechogenic lesions belong to the benign group (fig.5).

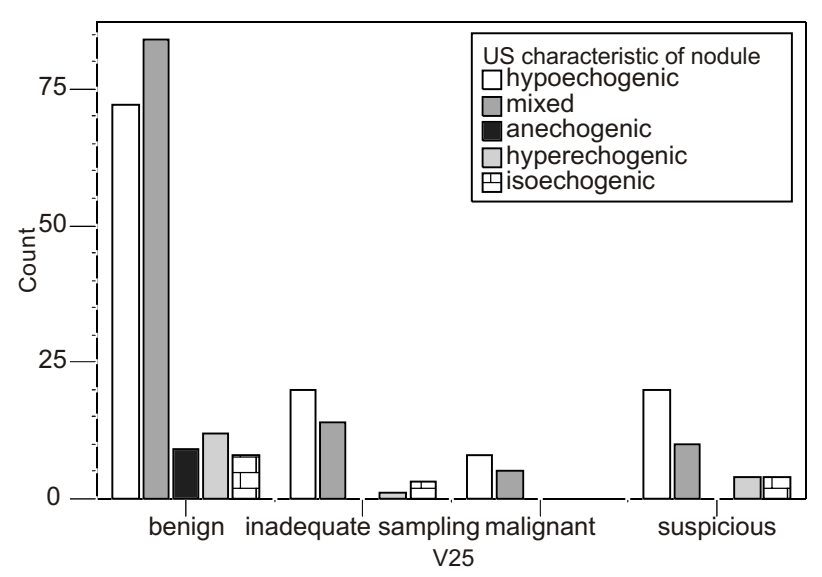

Fig. 5 Correlation between nodule echogenicity and cytological results

Table 1 illustrates the predictive value of some ultrasound characteristics. Hypoechogenicity, irregular margins, microcalcifications, intranodular vascularity and enlarged lymph nodes were defined as ultrasound criteria for malignancy and in $69,2 \%$ of the histologically proven malignant 
nodules three or more criteria were found (only a combination of these criteria is useful to predict malignancy - fig. 6). As shown on fig. 7 incidence of thyroid cancer was similar in solitary nodules $(4,96 \%)$ and in multinodular goiter $(4,37 \%)$.

Table 1. Ultrasound criteria for malignancy in different cytological groups of nodules

\begin{tabular}{||l|c|c|c||}
\hline \hline $\begin{array}{c}\text { US criteria for } \\
\text { malignancy }\end{array}$ & $\begin{array}{c}\text { Benign } \\
\text { lesions }\end{array}$ & $\begin{array}{c}\text { Suspicious } \\
\text { lesions }\end{array}$ & $\begin{array}{c}\text { Malignant } \\
\text { nodules }\end{array}$ \\
\hline $\begin{array}{l}\text { Solid hypoechogenic } \\
\text { pattern }\end{array}$ & $33,5 \%$ & $52,6 \%$ & $61,5 \%$ \\
\hline $\begin{array}{l}\text { Irregular (blurred) } \\
\text { margins }\end{array}$ & $7,0 \%$ & $18,4 \%$ & $69,1 \%$ \\
\hline Microcalcifications & $15,1 \%$ & $34,2 \%$ & $61,5 \%$ \\
\hline $\begin{array}{l}\text { Intranodular blood } \\
\text { flow }\end{array}$ & $12,9 \%$ & $10,5 \%$ & $50,8 \%$ \\
\hline $\begin{array}{l}\text { Enlarged lymph } \\
\text { nodes }\end{array}$ & $1,08 \%$ & $13,1 \%$ & $61,5 \%$ \\
\hline \hline
\end{tabular}
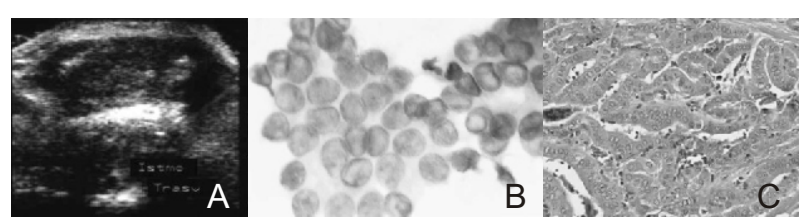

Fig. 6 (A-US, B-cytology, C-histology) illustrates a case of 60 years old man with a hard, rapidly growing nodule, hypoechogenic, with irregular margins and microcalcification. Cytology revealed papillary carcinoma. Histomorphological assessment confirmed the diagnosis.

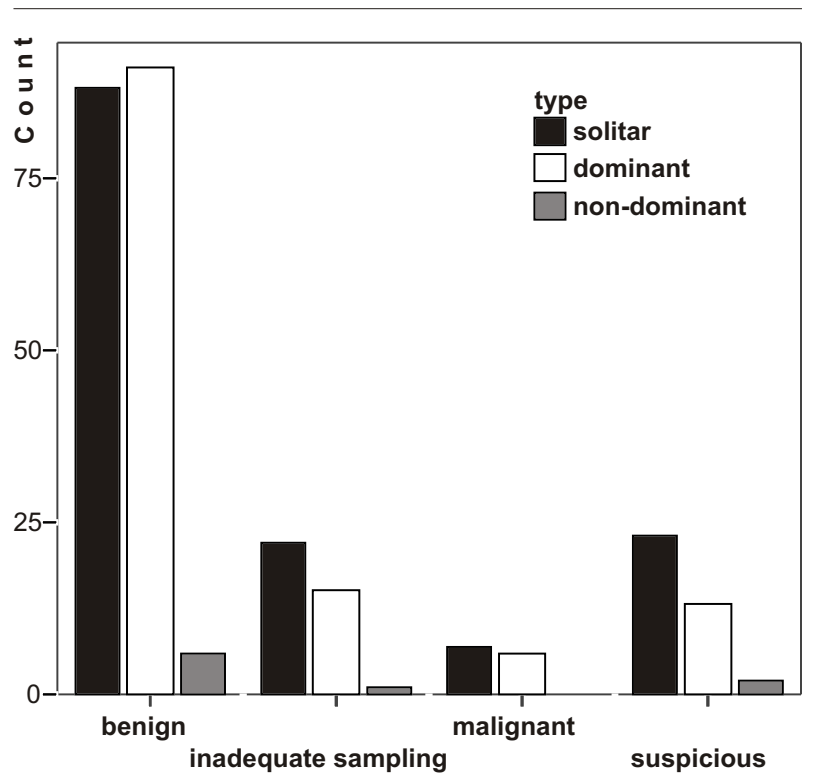

Fig. 7 Incidence of thyroid malignancy in solitary nodules and in dominant or non-dominant nodules of multinodular goiter

There was no correlation between nodule shape - oval or spherical and cytologically diagnosed malignant lesions (fig.8). According to our results the nodule shape may not serve as a predictor for malignancy.

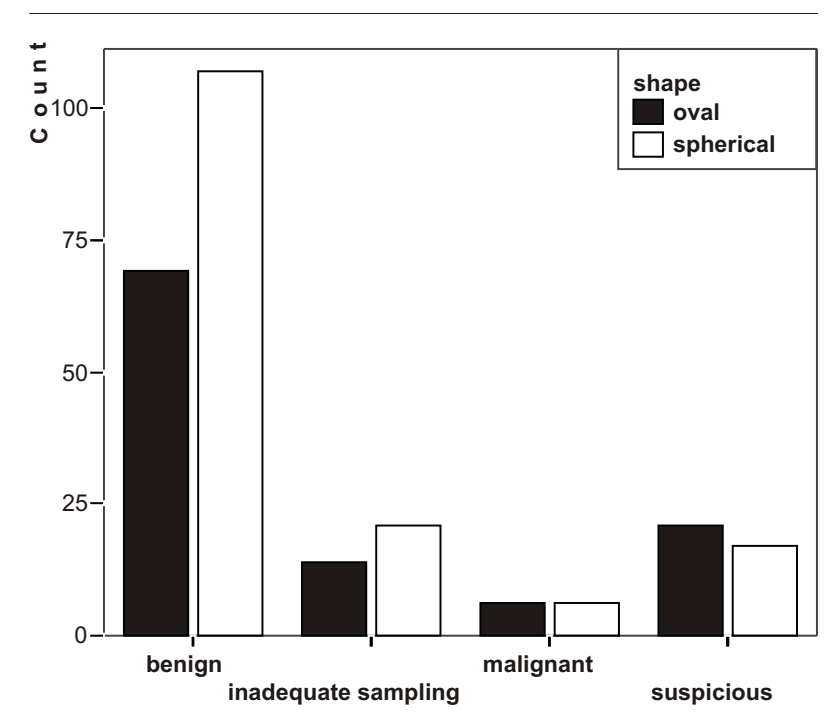

Fig. 8 Correlation between nodule shape (oval or spherical) and cytology

88 of the patients underwent thyroid surgery with histological examination. The comparison between cytological and histological findings showed that $99,1 \%$ of cytologically benign nodules were histologically confirmed, as well as $100 \%$ of the malignant lesions. $89,5 \%$ of the cases classified as suspicious on FNAB turned out to be benign on histology and 10,5\% were malignant. The discrepant cases were 2 false-negative results, which had a cytological diagnosis of nodular hyperplasia and turned out to be a medullary carcinoma.

\section{DISCUSSION}

Our results showed a sensitivity of $88,2 \%$ and a specifity of $100 \%$, positive predictive value $100 \%$, negative predictive value $91,3 \%$, accuracy $94,7 \%$.Limitations of FNAB are the inadequate samples and "indeterminate" diagnosis. We performed different techniques (US-guidence, FNAB without aspiration, multiple passes) in order to improve adequacy. It is important to avoid making a cytological diagnosis on a suboptimal sample. This was the reason for our two false negative cases - cytology of "nodular goiter", which turned out to be medullary carcinomas. Serum calcitonin is often elevated in cases of medullary carcinoma and should be measured in all patients with an "indeterminate" cytological diagnosis. The use of new, ancillary techniques, such as immunocytochemistry and RT-PCR may refine the diagnosis in "indeterminate" cases and improve the accuracy of FNAB.

\section{CONCLUSION}

The incidence of histologically proven thyroid cancer among all nodules is $4,7 \%$, whereas among the suspicious 
FNABs it is $10,5 \%$, which confirms the need for surgery in the latter group. Thyroid FNAB is an accurate and cost-effective preoperative tool for selection of patients who would benefit from surgery.

\section{REFERENCES}

1. Христозов К, И. Красналиев. Щитовидна жлеза в норма и патология, 2007

2. Belfiore A. The use of Fine Needle Aspiration Biopsy (FNAB) in thyroid disease. Thyroid International, 2002, /2/

3. Elisei R., V. Bottici, F. Fuchetti et al. Impact of routine measurement of serum calcitonin on the diagnosis and outcome of medullary thyroid cancer: experience in 10864 patients with nodular thyroid disorders. J Clin Endocrinol Metab 2004, Vol.89, 163-168

4. Gastro MR, Gharib H. Thyroid fine-needle aspiration biopsy: progress, practice, and pitfalls. Endocrine Practice 2003, 9, 128-136
5. Nguyen G.K., M.W. Lee, J. Ginnsberg, T. Wragg, D. Bilodeau. Fine-needle aspiration of the thyroid: an overview. CytoJournal 2005, 2:12

6. Pacini F, M Schlumberger, H Dralle, R Elisei, J W A Smit, W Wiersunga and European Thyroid Cancer Task force. European consensus for the management of patients with differentiated thyroid carcinoma of the follicular epithelium. Eur J Endocrinol 2006, 154: 787-803

7. Papini E., R. Guglielmi, A. Bianchini et al. Risk of malignancy in nonpalpable thyroid nodules: predictive value of Ultrasound and Color-Doppler features. J Clin Endocrinol Metab 2002, 87: 1941-1946

8. Saggiorato E., R.De Pompa, M. Volante et al. Characterization of thyroid "follicular neoplasms" in fine-needle aspiration cytological specimens using a panel of immunohistochemical markers: a proposal for clinical application. Endocrine-Related Cancer (2005), 12, 305-317 